\title{
Nanomedicine: from lab to industry
}

Volume I Issue 2 - 2014

\section{Editorial}

The magical word "Nano" has created a lot of excitement worldwide. Products of all types including sports wares, textiles, automobiles, satellites, medicines, etc, sound striking when prefaced with "Nano". The noun "nano" have started to sparkle in writing, reporting, and publishing and is been prefixed with all possible disciplines: chemistry, physics, electronics, photonics, mechanics, medicine, etc. Never in the history of science and technology there has been so much euphoria and excitement been created by the word "Nanotechnology". This euphoria has been prevailings so much that the policy makers have gone to the extent of recommending "Nano" as the solution to the overall problems of humanity. Ironically, to have a good chance of funding by science and technology agencies globally it is preferred to include the term "Nano" somewhere in the research proposals. This trend has been reflected in scientific community where too many new journals related to "nanotechnology" have started to appear. Each of these journals mostly has been interdisciplinary, giving an impression that science is no longer a one man show. Luckily, for me when I started my career in academics "nano" started expanding like the limitless universe. I had a unique opportunity to build a fast track career in this amazingly vibrant field of research that has been explosive growth. The potential of nano is yet to be tapped areas like medicine, agriculture, cosmetics, etc. However, many people (that also includes me) would debate that "nano" is not new at all. Before it became a catch phrase, people had already used nanomaterial for many centuries. As the ancient empires flaunted their impressive feats of engineering eg: Egyptian Pyramids, Greek Parthenons, and Indus valley to name a few, the craftsmen of those eras were also skilled at the opposite end of the spectrum at the nano level. The most notable examples in this regardare the Roman Lycurgus Cup and the Damascus swords. So does that mean that the engineers of that era were the only unknown benefactors of nanotechnology and other segments of society like health care practitioners did not gain? Well unlike engineering where the developments were based on sequential logical thoughts, medicine followed a holistic approach in most of the civilizations (i.e. with an insight of the mind, body and the spirit). The traditional medicinal practices such as Ayurveda seemed to have exploited nanotechnology unknowingly through this holistic approach. For instance, the use of herbo-mineral formulations of ayurveda constituting of 'bhasma'(residue after ritual incineration plant and animal by-product) and'pishti' (powdered gem or metal) were used for treatment of critical ailments and is well documented in ancient scriptures. Transmission electron microscopy has shown the presence of 50-150 nm gold and silver particles in these herbomineral formulations. Needless to say there are many examples which precedent the use of nanotechnology in ancient medicine. So it can be concluded that concepts that seeded nanotechnology (which were first discussed in 1959 by renowned physicist Richard Feynman in his famous talk "There's Plenty of Room at the Bottom") has not been a new thing in medicine. Just that we did not have means (characterization techniques) to explore it. Nanomedicine has become

\author{
Avinash Balakrishnan \\ Amrita Center for Nanosciences, India
}

Correspondence: Avinash Balakrishnan, Nanosolar Division (Center for Excellence), Amrita Center for Nanosciences, Ponekkara P.O. Kochi, Kerala, India, 68204I, Tel 9I-8I29777। I3, Email avinash.balakrishnan@gmail.com

Received: November 28, 2014 | Published: November 29, 2014

a booming industry where more than 1000 companies and 130 nanotech-based drugs worldwide have propped up. It is anticipated that a minimum of $\$ 4$ billion is being invested every year in this sector. As the industry continues to boom, it is expected to have a deep impact on the economy. Keeping up with this trend, research in nanomedicine has been moving ahead with tremendous pace. Each year, our inventory of materials for nanomedicine has been increasing enormously with a lot of work being published from different labs across the globe. Despite the many interesting results reported in the literature, there still exists a big void that prevents many of these nanomaterials to be translated from mere lab studies to industrial production. The problem mainly lies with scalability. It is at times not feasible to scale up the production of many of these nanocrystals with controlled morphology and sizes by simply increasing the size and amount of the reactors and reagents, respectively. Even if this scalability issue is addressed, maintaining the high quality of the desired products at low cost becomes a challenge. No doubt the issues related to toxicity and environmental impact of nanoscale materials needs to be also taken into consideration. But these challenges have not stopped researchers from functionalizing nanomaterials with biomolecules or structures making it useful for both in vivo and in vitro biomedical applications. This integration materialscience with biology at nanoscale has led to the development of diagnostic tools, imaging kits, biomarkers, analytical tools, etc. The national nanotechnology initiatives taken up worldwide expectto drive these new commercial applications in the pharmaceutical sector. As our capabilities for fabricating nanomaterials progressively continues, we should always try to channelize our efforts into commercial applications. The purpose of "Journal of Nanomedicine Research" is to present a series of articles and reviews by the leading experts worldwide who can provide insights on the recent developments in nanomaterials. The idea is to the acquaint readers with the multiple facets of nanomaterials in medicine and biomedical related areas. We hope that all readers will find that the work presented in the journal beneficial to their existing research and will begin to explore into this exciting field of nanomedicine if they have not already done so.

\section{Acknowledgments}

None.

\section{Conflicts of interest}

None. 